\title{
Helicobacter pylori: 20 años después
}

\author{
Helicobacter pylori: \\ Two decades later
}

\begin{abstract}
Twenty years ago Helicobacter pylori was identified in the gastric content and this finding stimulated a multitude of studies and publications that changed drastically our knowledge of common gastric conditions such as peptic ulcer and gastric cancer. This progress has affected significantly our practice and has resulted in great benefits for our patients. The relationship of the bacteria to the pathogenesis of gastritis, peptic ulcer, gastric cancer and gastric lymphoma was firmly established. A large number of individuals are infected (an estimated half of the world's population) while only a minority suffers of the above mentioned conditions, so it became clear that certain strains of the bacteria, susceptibility of the host and environmental factors interplay in causing the damage. The diagnosis of the infection became a standard practice and therapy and prevention of these common conditions has been drastically modified. Eradication of the bacteria resulted in the cure of ulcers, in a dramatic decrease in the rate of its complications and in the ocurrence of gastric cancer. Better sanitary conditions are part of the solution. Also it is successful in the management of certain gastric lymphomas. The treatment however, is expensive and not free of side effects. Further research work is needed to facilitate the diagnosis and less expensive treatments should be available to eradicate Helicobacter pylori throughout the world (Rev Méd Chile 2004; 132: 1339-44).
\end{abstract}

(Key Words: Gastritis; Helicobacter pylori; Peptic ulcer; Stomach neoplasms)

$\mathrm{H}$ an pasado dos décadas, desde que el Helicobacter pylori fue cultivado a partir de muestras de contenido gástrico y ello dio origen a numerosos estudios que han precisado su papel patogénico en las enfermedades gastrointestinales. Cabe reflexionar ¿qué progreso ha habido en nuestro conocimiento sobre esta bacteria, qué ha significado ello para nuestra práctica médica $\mathrm{y}$ qué beneficio ha brindado a nuestros enfermos?

1) Epidemiología: Se ha determinado que aproximadamente la mitad de la población mundial está infectada con la bacteria $\mathrm{H}$ pylori, con mayor prevalencia en los países en vías de desarrollo y estrechamente en relación con los niveles socioeconómicos. Si bien la mayoría de estos pacientes infectados permanecen prácticamente asintomáticos por el resto de sus vidas, hay evidencias sólidas de que el $\mathrm{H}$ pylori tiene un papel patogénico importante en ciertas enfermedades digestivas (Tabla 1).

2) Fisiopatología: La patología gastroduodenal que puede desarrollar la infección por $\mathrm{H}$ pylori en cada paciente está determinado por la interacción de: a) la virulencia de la bacteria; b) la respuesta inmunológica del huésped; c) la convergencia de ambos factores, que puede ser influenciada por condiciones ambientales tales como la infección temprana en la vida, desnutrición, ingesta de compuestos nitrosos, déficit en vitamina $C$, tabaquismo, etc. 


\section{Tabla 1. Patología digestiva relacionada con el H pylori}

- Gastritis aguda y crónica, antral, corporal y atrofia gástrica, que puede evolucionar a la metaplasia intestinal.

- Ulcera péptica, tanto gástrica como duodenal y relacionada con la gastritis crónica.

- Adenocarcinoma gástrico distal, también relacionado con la gastritis.

- Linfoma de tipo MALT de bajo grado.

- Posiblemente, en un grupo de pacientes con gastritis y dispepsia pseudo-ulcerosa.

El estómago, por el bajo pH de su mucosa, tiene una importante actividad bactericida, sin embargo el $\mathrm{H}$ pylori tiene diversos mecanismos que le permiten evadir esta actividad, colonizar la mucosa gástrica y establecer una permanencia de largo plazo. La bacteria tiene flagelos que le permiten penetrar y anidarse bajo la capa de mucus, la ureasa que le crea un nicho neutro protector y la adhesión a las células epiteliales mediada por adhesinas (BabA) que se unen al grupo sanguíneo Lewis B.

Ha habido un enorme progreso en el conocimiento de las características genómicas de la bacteria, aunque debemos admitir que este progreso no se ha reflejado total y directamente en aplicaciones clínicas. La secuencia genómica completa de la bacteria se identificó en la última década y se han clarificado los mecanismos de citotoxicidad bacteriana, representada especialmente, cuando están presentes los genes CagA, VacA y del islote de patogenicidad, que se traslocan a la célula epitelial del huésped, producen una activación de los neutrófilos y generan un aumento en la producción de interleuquinas IL-8, IL-1ß, IL-2 y IL-6 1 .

La importancia de los genes VacA y de sus genotipos s2/m2, s1/m1, s1/m2; del gen CagA y del gen iceA tipo A2, en la inducción de daño epitelial, aparece enfatizada por los estudios de Araya y cols, comunicados en este número de la Revista (Rev Méd Chile 2004; 132: 1545-54) ${ }^{2}$.

Una revisión somera del proceso de infección puede ser:

I) Fase aguda de la infección: Se adquiere principalmente por vía oral, destacándose la transmisión por los miembros de la familia (lo que es más evidente cuando hay hacinamiento) y por vectores, como las moscas, que abundan especialmente cuando hay malas condiciones higiénicas y socioeconómicas.

II) Fase crónica: con el tiempo se desarrolla una respuesta linfocitaria adaptativa, facilitada por la expresión de adhesinas vasculares (VCAM-1 y ICAM-1) que facilitan la migración de los macrófagos a la mucosa gástrica. Los macrófagos, a su vez, desencadenan una cascada inflamatoria que determinará, con el tiempo, el tipo y gravedad de la gastritis. Los linfocitos de tipo 〈helper» modifican la inflamación; en este caso predomina el tipo Th1 (con liberación de IL-2 e INF- $\gamma$ ). A su vez el INF- $\gamma$ induce la expresión de complejos de histocompatibilidad (MHC). Otra citotoxina bacteriana, la vacuolizante VacA, induce vacuolización del epitelio y apoptosis que es mediada por el factor de necrosis tumoral (FNT- $\alpha$ ), lo que resulta en la destrucción lenta y progresiva de la barrera epitelial. A este último fenómeno contribuyen citoquinas producidas por los macrófagos. La reacción inflamatoria con activación de los neutrófilos resulta en una mayor producción local de COX-2 e iNOS. El polimorfismo proinflamatorio de la IL-1ß favorece el desarrollo de gastritis predominantemente corporal, con atrofia e hipoclorhidria, mientras que la ausencia de este polimorfismo causa más bien gastritis antral, que rara vez lleva a la gastritis corporal y fúndica, y está más a menudo asociada a úlcera duodenal. Algunas de las citoquinas producidas por la interacción de la bacteria y el epitelio alteran directamente la secreción ácida por las células parietales. En este sentido el FNT- $\alpha$, la 
IL-1ß y el INF- $\gamma$ aumentan la entrega de gastrina por las células $\mathrm{G}$ antrales y causan una disminución de las células $\mathrm{D}$ que producen somatostatina, inhibidor de las células parietales, lo que resulta en un aumento neto en la secreción de ácido. Finalmente, el huésped produce IgA e IgG anti $\mathrm{H}$ pylori, que si bien no erradican la bacteria, pueden aumentar el daño tisular y su determinación en la sangre puede tener utilidad diagnóstica ${ }^{1}$.

La interacción entre la bacteria, la respuesta inmunológica y factores ambientales determinará dos tipos de gastritis: una, que compromete principalmente el antro gástrico, con hipersecreción ácida y asociada más frecuentemente a la úlcera duodenal o pilórica, y otra, con compromiso más proximal, que compromete el cuerpo y fondo gástricos, con predominio de atrofia de la mucosa, metaplasia intestinal y asociado más a menudo a cáncer gástrico. La sola presencia de los factores CagA y VacA no explica el curso distinto que pueden seguir los pacientes infectados hacia un determinado tipo de gastritis. La genética del huésped, entre ellos los alelos polimórficos IL-1ß-31 e IL-1RN se asocian a mayores niveles de las citoquinas proinflamatorias IL-1ß y FNT- $\alpha$ que, entre otras acciones, inhiben la secreción ácida y de pepsina ${ }^{3}$.

III) H pylori y secreción gástrica de ácido: El efecto de la infección por $\mathrm{H}$ pylori sobre la secreción gástrica es multifactorial y determinado por: a) Inicialmente la infección disminuye la producción de ácido por acción de citoquinas como la IL-1ß. b) Posteriormente la secreción de ácido está determinada por la severidad y la localización de la gastritis, determinada según se vio por los alelos IL-1ß y IL-1RN ${ }^{4}$. Los pacientes con gastritis localizada en el antro son hipersecretores, un efecto mediado en parte por la IL-8, lo que resulta en una mayor producción de gastrina y menor entrega de somatostatina antral. Cuando la gastritis compromete el cuerpo hay hipoacidez y, eventualmente, anacidez cuando se llega a la atrofia. Un anticuerpo contra la $\mathrm{H}+\mathrm{K}+$ /ATPasa de las células parietales puede contribuir a disminuir la secreción ácida y la producción de gastritis atrófica.
3) H pylori y úlcera péptica: El papel relevante del $\mathrm{H}$ pylori en la patogénesis de la gastritis y la úlcera péptica, tanto gástrica como duodenal, es sustentado por numerosas experiencias que demuestran que la erradicación del $\mathrm{H}$ pylori puede revertir la gastritis, si ésta no ha llegado a un estado muy avanzado de atrofia gástrica $\mathrm{y}$, prácticamente, elimina las recurrencias de la enfermedad ulcerosa, a menos que intervengan otros factores como es el uso de antiinflamatorios no esteroidales (AINEs) y la hipersecreción gástrica que puede asociarse a gastrinomas ${ }^{5}$. Como resultado, la incidencia de úlceras pépticas ha disminuido francamente y las complicaciones de la úlcera, como son la perforación, la obstrucción pilórica y tratamiento quirúrgico de la úlcera son cada vez más infrecuentes.

4) H pylori y cáncer gástrico: La asociación patogénica entre el cáncer gástrico y la infección por H pylori ha sido reconocida desde 1994, cuando la OMS lo declaró cancerígeno de tipo I. La infección por $\mathrm{H}$ pylori se relaciona con dos tipos de cáncer gástrico: el de tipo difuso y el de tipo intestinal. En esta última variedad, se ha postulado una progresión de la gastritis superficial a la atrofia y a la metaplasia intestinal. Interesantes publicaciones, más recientes, han proporcionado evidencias adicionales para reforzar la relación entre la infección por $\mathrm{H}$ pylori (HP) y el cáncer gástrico. Uemura et $\mathrm{al}^{6}$ observaron que $2,9 \%$ de 1.526 pacientes en quienes había serología HP (+) previamente, y fueron seguidos por 8 años, desarrollaron cáncer gástrico, el que por el contrario no se detectó en los otros pacientes que eran HP (-). En esa misma publicación, entre 253 pacientes que eran previamente HP (+) y fueron previamente erradicados, ninguno de ellos desarrolló cáncer gástrico en el seguimiento. Similares experiencias han sido reportadas en China ${ }^{7}$. Más aún, Correa et al observaron que la erradicación del $\mathrm{H}$ pylori con un suplemento dietético con antioxidantes, favoreció la regresión de lesiones preneoplásicas como son la atrofia gástrica y la metaplasia intestinal en una región de alto riesgo de cáncer gástrico en Sudamérica ${ }^{8}$. En el mismo sentido, se ha observado que parientes de pacientes con cáncer gástrico tienen un aumento de lesiones precancerosas, las que ocurren sólo en aquellos que son HP (+), a la vez que se ha observado que la prevalencia de 
infección por HP es mayor en sujetos con antecedentes familiares de cáncer gástrico.

Se ha observado una disminución del cáncer gástrico en varios países, lo que puede haber sido determinado por la erradicación de la bacteria y también por la mejoría en los factores socioeconómicos con mejor higiene, alimentación y disponibilidad de agua potable, con disminución en la frecuencia de la infección. Basados en todo lo anterior podemos asumir que si se dispusiera de un método eficaz y económico de erradicación del $\mathrm{H}$ pylori podríamos anticipar una mayor disminución y eventual desaparición del cáncer gástrico.

5) $\mathrm{H}$ pylori y linfomas de la mucosa gástrica asociados a tejido linfoide (MALT): El $\mathrm{H}$ pylori tiene un papel patogénico en el linfoma que se relaciona con el aumento en la presencia de células plasmáticas y de los linfocitos de la mucosa, los que progresivamente se organizan en folículos linfáticos, con centros germinales y zonas en mantel. Estos folículos pueden crecer, hacerse prominentes con células $\mathrm{B}$ en la periferia $\mathrm{y}$ pueden evolucionar, bajo estímulo de linfocitos T, que a su vez están estimulados por el $\mathrm{H}$ pylori, en poblaciones monoclonales que crecen y forman un linfoma. Por un cierto tiempo, la sobrevida de estos linfomas está condicionada por la presencia de células $\mathrm{T}$ sensibilizadas por el $\mathrm{H}$ pylori. En esta etapa, el linfoma puede curarse erradicando la bacteria. En una etapa más avanzada la proliferación linfoide se independiza, los linfomas crecen lentamente, diferenciados, pero algunos pueden desdiferenciarse y formar neoplasias malignas que se diseminan localmente y a distancia.

En la práctica se ha observado que aproximadamente $70 \%$ de los pacientes infectados con $\mathrm{H}$ pylori, y a quienes se les diagnostica un linfoma de tipo MALT, la erradicación del $\mathrm{H}$ pylori resulta en desaparición del linfoma y de las células B de la mucosa gástrica.

6) $\mathrm{H}$ pylori y esofagitis por reflujo gastroesofágico (ERGE): Este es un tema controversial y no totalmente resuelto y puede estar determinado por el tipo de población estudiada, por el tipo de gastritis, antral o corporal, asociada a la infección por $\mathrm{H}$ pylori $\mathrm{y}$, a su vez, al efecto sobre la secreción gástrica de ácido.
Diversos grupos reportaron un aumento en la incidencia de ERGE en ulcerosos duodenales tratados con erradicación del $\mathrm{H}$ pylori, mientras otros sugirieron que el manejo de la ERGE se puede hacer más difícil después de erradicar el $\mathrm{H}$ pylori. Se podría postular que el $\mathrm{H}$ pylori tendría un papel protector de la ERGE al producir una gastritis de la mucosa parietal, hiposecreción ácida, a la vez que por la producción de amonio potencia la terapia inhibidora de ácido de los antisecretores. En apoyo de esta hipótesis, se ha comunicado que pacientes con esófago de Barrett tienen una tasa de infección por H pylori significativamente menor que otros enfermos sin Barrett ${ }^{9}$. Se cuestionaría entonces si debe erradicarse el $\mathrm{H}$ pylori en pacientes que sufren ERGE. Por el contrario, se puede argumentar que en ciertos pacientes con ERGE hay una gastritis con tendencia a hipersecretar ácido, que es similar a lo que ocurre en ulcerosos duodenales y que la erradicación del $\mathrm{H}$ pylori no aumenta la secreción ácida y a la larga no exacerba la ERGE. Además, hay evidencias que la erradicación del $\mathrm{H}$ pylori disminuye marginalmente la acción inhibidora de la secreción ácida de los inhibidores de la bomba de protones, lo que no influye significativamente en el efecto de estos inhibidores sobre la esofagitis. El resultado de la erradicación estará determinado según la población estudiada. En casos con gastritis antral e hipersecreción, la erradicación del $\mathrm{H}$ pylori no favorecería la aparición o agravación de la ERGE mientras que en los portadores de gastritis predominantemente corporal este factor no jugaría un papel decisivo ${ }^{10}$. Por otra parte, parece haber una opinión mayoritaria a favor de erradicar el $\mathrm{H}$ pylori en pacientes con ERGE, especialmente en países con alta incidencia de cáncer gástrico (con gastritis corporal), en quienes hay mayor beneficio en la erradicación, comparado con la posible disminución de la eficacia de la terapia anti ERGE, incluso en aquellos que van a ser sometidos a terapia con inhibidores de la bomba de protones (IBP) por largo tiempo.

7) Diagnóstico de la infección por H pylori: Los métodos diagnósticos han sido evaluados en diversas publicaciones ${ }^{1}$ y la sensibilidad y especificidad de ellos están resumidas en la Tabla 2. La elección en cada centro de un método diagnóstico estará determinado por su costo y disponibilidad, 
Tabla 2. M étodos diagnósticos de la infección por H pylori

\begin{tabular}{|lccc|}
\hline & Sensibilidad (\%) & Especificidad (\%) & Costo \\
\hline Biopsia de la mucosa* & $79-100$ & $90-100$ & + \\
Urea marcada C13 o C14 (aliento) & $>90$ & $>90$ & ++ \\
Serología (IgG) & $>90$ & $>90$ & + \\
Antígeno en las heces & $89-98$ & $>90$ & + \\
Histología & 90 & $>90$ & +++ \\
\hline
\end{tabular}

*= Si está indicada la endoscopia.

tomando en consideración la necesidad de indicar endoscopia.

El test de ureasa en la biopsia de mucosa y el del aire espirado pueden ser falsamente negativos si hay uso reciente de antibióticos, sangrado gástrico o uso concomitante de inhibidores de la secreción ácida.

8) Erradicación del H pylori: En todas las condiciones mencionadas en la Tabla 1 hay clara indicación de erradicación. La enfermedad ulcerosa activa 0 por antecedentes, especialmente cuando hay o ha habido complicaciones, pacientes que necesitan ingerir frecuentemente AINEs, especialmente si han sangrado, casos de cáncer gástrico precoz tratados quirúrgicamente o por endoscopia y parientes de primer grado de pacientes con cáncer gástrico.

También está indicado en pacientes con ERGE que precisan de IBP a largo plazo. No hay consenso entre los distintos autores si el $\mathrm{H}$ pylori debe erradicarse en pacientes con dispepsia pseudo ulcerosa, habiendo sólo un grupo pequeño que se beneficiaría.

La terapia ideal para erradicar el $\mathrm{H}$ pylori debe ser eficaz, fácil de administrar, barata, que no induzca resistencia y que esté libre de efectos secundarios. Esta terapia no ha sido encontrada aún. Numerosos ensayos clínicos, en varios miles de pacientes en muchos países en el mundo ${ }^{1}$, han demostrado que los pilares del tratamiento son: Una fuerte inhibición de la secreción ácida, ya sea con IBP o altas dosis de antagonistas H2 de la histamina, administrando al mismo tiempo dos antibióticos, uno de los cuales pueden ser las sales de bismuto. La combinación de antibióticos puede variar según costo, tolerancia y disponibilidad en cada país, siendo señalable que en Latinoamérica el $\mathrm{H}$ pylori tiene una alta resistencia al metronidazol ${ }^{5}$. La combinación de antibióticos administrada por 7 a 14 días produce una erradicación de aproximadamente $80 \%$ con aceptable frecuencia de efectos secundarios. Se han reportado variantes con menos días de tratamiento y con similares resultados. El costo de esta terapia varía entre los países pero no es menor y deja al margen a un alto porcentaje de la población mundial de bajos recursos, que paradojalmente es la que más necesita la erradicación ${ }^{5}$. Una vacuna está siendo activamente estudiada, pero aún no hay resultados concretos.

Para concluir, en estos 20 años, como consecuencia de la información acumulada en relación con la infección por $\mathrm{H}$ pylori, ha ocurrido un cambio fundamental en nuestro enfoque de patologías digestivas frecuentes como son la gastritis crónica y sus complicaciones, la enfermedad ulcerosa y el cáncer gástrico. Estas condiciones patológicas han pasado a pertenecer a la categoría de enfermedades infecciosas tratables y la erradicación de la bacteria cambia radicalmente el curso de la úlcera, la frecuencia de sus complicaciones y la incidencia de cáncer gástrico. Desafortunadamente, la erradicación sigue siendo compleja, de alto costo y no exenta de efectos colaterales. El desarrollo de una vacuna que erradique o prevenga la infección podría convertir a ambas enfermedades en entidades de curiosidad histórica.

Jorge Valenzuela $E$.

Profesor de Medicina, Universidad de Chile. Clínica Las Condes, Santiago de Chile. 


\section{REFERENCIAS}

1. Suerbaum S, Michetti P. Helicobacter pylori infection. N Engl J Med 2002; 347: 1175-86.

2. Araya JC, Anabalón L, Roa I, Bravo M, Viшaseca Ma, GuZMán P ET AL. Relación de la genotipicación de Helicobacter pylori con la forma e intensidad de la gastritis en población adulta portadora de patología gástrica benigna. Rev Méd Chile; 2004; 132: 1345-54.

3. Queiroz DMM, Mendes EN, Rocha GA, Ouveira CA, Magalhaes PP, Moura SB et al. CagA positive Helicobacter pylori and risk for developing gastric carcinoma in Brazil. Int J Cancer 1998; 78: 135-9.

4. Queiroz DMM, Guerra JB, Rocha GA, Rocha AMC, Santoa A, Oliveira AG et al. IL-1ß and IL-1RN Polymorphic Genes and Helicobacter Pylori cagA Strains Decrease the Risk of Reflux esophagitis. Gastroenterology 2004; 127: 73-9.

5. Coelho LG, Leon-Barua R, Quigley EM. LatinAmerican Consensus Conference on Helicobacter pylori infection. Am J Gastroenterol 2000; 95: 2688-91.
6. Uemura $\mathrm{N}$, Окамото S, Yамamoto S, Matsumura $\mathrm{N}$, YAMAGUCHI S, YaMAKIDO $M$ ET aL. Helicobacter infection and the development of gastric cancer. N Engl J Med 2001; 345: 784-9.

7. Wong BC, Lam SK, Wong WM, Chen JS, Zheng TT, FENG RE ET AL. Helicobacter pylori eradication to prevent gastric cancer in a high risk region of China. JAMA 2004; 291: 187-94.

8. Correa P, Fontham ETH, Bravo JC, Bravo LE, Ruiz B, ZaRAMA G ET AL. Chemoprevention of gastric dysplasia: randomized trial of antioxidant supplements and anti-helicobacter pylori therapy. J Nat Cancer Inst 2000; 92: 1881-8.

9. Abe O, Koike T, Sekine H, Ijjima K, Kawamura M, ImATANi A ET AL. The prevalence of Helicobacter pylori infection and the status of gastric acid secretion in patients with Barrett's Esophagus. Am J Gastroenterol 2004; 99: 121321.

10. Pandolfino Je, Howden CW, Kahrilas PJ. H pylori and GERD: Is less more? Am J Gastroenterol 2004; 99: 1222-5. 